\title{
Modelling the Rayleigh match
}

\author{
P.B.M. THOMAS AND J.D. MOLLON \\ Department of Experimental Psychology, University of Cambridge, Cambridge CB2 3EB, UK \\ (Received September 7, 2003; AccePted February 18, 2004)
}

\begin{abstract}
We use the photopigment template of Baylor et al. (1987) to define the set of Rayleigh matches that would be satisfied by a photopigment having a given wavelength of peak sensitivity $\left(\lambda_{\max }\right)$ and a given optical density (OD). For an observer with two photopigments in the region of the Rayleigh primaries, the observer's unique match is defined by the intersection of the sets of matches that satisfy the individual pigments. The use of a template allows us to illustrate the general behavior of Rayleigh matches as the absorption spectra of the underlying spectra are altered. In a plot of the $Y$ setting against the red-green ratio (R), both an increase in $\lambda_{\max }$ and an increase in optical density lead to an anticlockwise rotation of the locus of the matches satisfied by a given pigment. Since both these factors affect the match, it is not possible to reverse the analysis and define uniquely the photopigments corresponding to a specific Rayleigh match. However, a way to constrain the set of candidate photopigments would be to determine the trajectory of the change of match as the effective optical density is altered (by, say, bleaching or field size).
\end{abstract}

Keywords: Rayleigh match, Model, Optical density, Anomalous trichromacy, Pseudoprotanomaly

\section{Introduction}

The Rayleigh match (Rayleigh, 1881) is a traditional tool for classifying color vision. Typically, an observer is shown a circular, bipartite field of less than 2-deg diameter. One half-field contains a near-monochromatic yellow light $(c a .590 \mathrm{~nm})$, while the other contains a mixture of red $(c a .679 \mathrm{~nm})$ and green $(c a .545 \mathrm{~nm})$ light. The observer adjusts the intensity of the yellow field, and the relative amounts of red and green in the mixture field, in order to make the two half-fields appear identical. Three features of the match are of interest: the match midpoint (i.e. the composition of the mixing field), the level of the reference yellow, and the range of mixtures accepted.

According to standard theory, each class of retinal photoreceptor obeys the Principle of Univariance (Rushton, 1972), and the observer accepts the Rayleigh match when the two half-fields produce the same set of quantum catches at the level of the photoreceptors. Since the short-wave cones are insensitive to the Rayleigh primaries, the trichromatic observer equates quantum catches for two classes of cone, whereas the dichromatic observer need satisfy only one class.

The match will thus be affected by any factor that alters the sensitivities of the photopigments to the three primaries of the Rayleigh match. The most prominent such factors are the wavelength of peak sensitivity $\left(\lambda_{\max }\right)$ of each photopigment and the optical density of each photopigment (Mitchell \& Rushton, 1971a;

Address correspondence and reprint requests to: Peter B.M. Thomas, Department of Experimental Psychology, University of Cambridge, Downing Street, Cambridge, CB2 3EB, UK. E-mail: pbmt2@cam.ac.uk
Smith et al., 1978; He \& Shevell, 1995). Variations in optical density, for example, have been used to model the effects on the Rayleigh match of bleaching (Baker, 1966; Alpern, 1979), of field size (Pokorny \& Smith, 1976), and of macular disorders that alter the alignment of photoreceptors (Pokorny et al., 1978).

The action spectra of primate cones have been recorded by the suction-pipette technique, and it appears that the absorbance spectra of opsins of different $\lambda_{\max }$ can all be represented by the same fixed template if the spectra are plotted on an abscissa of log wavenumber (Baylor et al., 1987). Baylor and his colleagues define their template with a polynomial function. In the present study, we take advantage of this polynomial to model the general behavior of the Rayleigh match as the $\lambda_{\max }$ and the optical density of the underlying pigments are systematically varied. For pigments of given $\lambda_{\max }$ values and given optical density, a unique Rayleigh match can be estimated. Conversely, for a given Rayleigh match, the model allows a search for combinations of $\lambda_{\max }$ and optical density that are compatible with the match. We modelled the matching behavior expected for a particular clinical instrument, the Oculus anomaloscope. The Oculus, which uses light-emitting diodes as sources, is designed to simulate the classical Nagel anomaloscope.

\section{Materials and methods}

The spectral power distributions of the primaries of an HMC anomaloscope (Oculus Optikgeräte $\mathrm{GmbH}$, Germany) were measured using a spectroradiometer (PhotoResearch 650, Chatsworth, CA). Since the outputs of each primary were found to vary near linearly with scale setting, the emission spectra of the primaries at 
maximum output could be scaled to generate the spectral output at any particular setting on the anomaloscope. The reference light $(Y)$ scales in intensity from 0 to 45 . The mixing light $(R)$ is a mixture of the red and green primaries, where a value of $R=0$ means that the mixture is entirely composed of the green primary and a value of $R=73$ means the mixture is entirely composed of the red primary.

The absorption spectra of hypothetical photopigments were generated with the template of Baylor et al. (1987) and were corrected for the effects of self-screening (Brindley, 1953; Alpern et al., 1987). The effect of macular pigment (Bone et al., 1992) was not taken into account since the Rayleigh primaries lie at wavelengths above those filtered significantly by the macular pigment. There is a small filtering effect of the lens in the region of the green primary (Stockman et al., 1999); and so this effect was included in the model.

Once a corrected absorption spectrum $(S \lambda)$ had been generated for a pigment of given $\lambda_{\max }$ and optical density, it was multiplied by the spectral power distribution of the $G$ primary $(G \lambda)$, so as to give the total excitation $\left(E_{G}\right)$ of the pigment for a setting of $R=0$. The absorption spectrum $(S \lambda)$ was multiplied similarly by the spectral power distribution of the yellow primary $(Y \lambda)$, to give the total excitation $\left(E_{Y}\right)$ of the pigment for the maximal setting of $Y$. The value $45\left(E_{G}\right) /\left(E_{Y}\right)$ then gives the setting of $Y$ needed to equate the absorptions in the two half-fields. An equivalent calculation gives the setting of $Y$ needed to match the excitation produced by the $R$ primary. Since the anomaloscope scales are linear, these two matches, at the endpoints of the red-green mixture scale, define the settings of $Y$ needed at intermediate values of $R$. In the traditional plot of $Y$ against red-green ratio (e.g. Fig. 1), the set of matches that satisfy a given pigment fall on a straight line. This matching locus for a single photopigment of absorption spectrum $S(\lambda)$ thus comprises the solutions for $R, G$, and $Y$ of

$$
\int Y(\lambda) S(\lambda) \mathrm{d} \lambda=\int R(\lambda) S(\lambda) \mathrm{d} \lambda+\int G(\lambda) S(\lambda) \mathrm{d} \lambda
$$

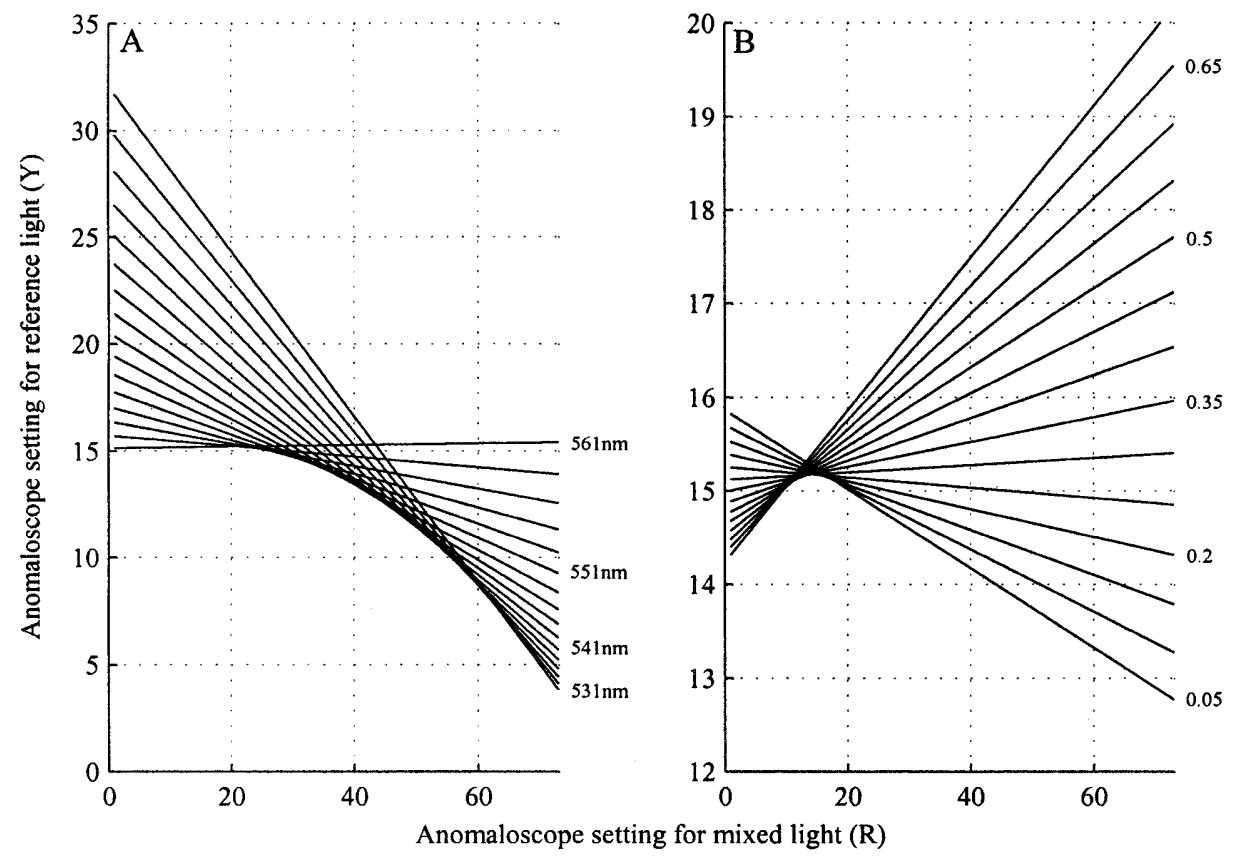

If now a second absorption spectrum is derived from the Baylor polynomial, a new locus can be generated, representing the combinations of $Y$ and $R$ that would give matches satisfying the second pigment. The point in the diagram where the first and second loci intersect must represent the unique values of $Y$ and $R$ that equate the quantum catches for both the hypothetical pigments. This point is the theoretical Rayleigh match of an observer whose retina contains the two pigments.

To determine the photopigments satisfied by a particular Rayleigh match, candidate photopigments were generated with peak sensitivity in a given range, usually $500-600 \mathrm{~nm}$, at a series of optical densities. The excitation caused by the reference and mixing lights was calculated by scaling the anomaloscope light emission spectra by the values of the match, and multiplying these spectra by the absorption spectra generated for the candidate photopigments. Photoreceptors in which the reference and mixing lights produce the same level of excitation are those satisfied by the Rayleigh match.

Our modelling assumes that match midpoints are unaffected by the relative numbers of different cone types or by postreceptoral processes. Such factors may, of course, affect the range of matches accepted by the observer.

\section{Results}

\section{Determination of the Rayleigh matches that satisfy particular pigments}

Fig. 1A shows the loci of Rayleigh matches that will be accepted by photopigments at a range of peak sensitivities. In this plot, optical density is held constant at 0.3 . Conversely, Fig. 1B shows the loci of Rayleigh matches for a range of values of optical density and a fixed $\lambda_{\max }$ of $561 \mathrm{~nm}$. It can be seen that either an increase in $\lambda_{\max }$ or an increase in optical density leads to an anticlockwise rotation of the locus of accepted matches. This is a general rule for pigments with a $\lambda_{\max }$ in the range $530-570 \mathrm{~nm}$ and
Fig. 1. Effects of $\lambda_{\max }$ and optical density on matching locus. Fig. 1A shows the anticlockwise rotation caused by increasing peak sensitivity. Fig. 1B shows the anticlockwise rotation caused by increasing optical density. 
for optical densities in the range $0-0.6$. However, the rotations are not simple rotations around a single point.

Since each of the individual loci in Fig. 1 represents the set of matches that satisfy a single pigment, such a locus shows the set of matches predicted for a hypothetical dichromat who is limited to this one photopigment in the region of the Rayleigh primaries (Mitchell \& Rushton, 1971b; Alpern \& Wake, 1977): by adjusting only the setting of $Y$, the dichromat can match any setting of $R$. If a $\lambda_{\max }$ of $534 \mathrm{~nm}$ and an optical density of 0.3 are assumed, the model gives a locus resembling the loci classically generated by protanopes, while a $\lambda_{\max }$ of $561 \mathrm{~nm}$ and an optical density of 0.3 gives a classically deuteranopic locus.

For an observer with two pigments in the range of the Rayleigh primaries, the predicted match midpoint is determined by the intersection of the loci corresponding to the individual pigments: only at this point can the quantum catches be equated in both pigments. We may expect that the matching range will depend on the angle of intersection of the two loci, becoming narrower the larger the angle of intersection.

Modelling of pigments with $\lambda_{\max }$ at 534 and $561 \mathrm{~nm}$, with optical density set at 0.3 , yields a match at $R=40.8$ and $Y=15.3$, close to the nominal setting of the normal observer $(R=40$, $Y=15$ ). If the $\lambda_{\max }$ of the long-wave pigment is taken to lie at a slightly shorter wavelength, as is thought to occur when alanine replaces serine at site 180 in the amino acid sequence (Winderickx et al., 1993), the locus for the pigment undergoes a small clockwise rotation. For a 4-nm shift (Merbs \& Nathans, 1992), the intersection occurs at $R=44.4$ and $Y=14.1$.

If protanomaly is taken to depend on two variant forms of the middle-wave pigments $\left(\mathrm{M}, \mathrm{M}^{\prime}\right)$ and deuteranomaly to depend on two forms of the long-wave pigment $\left(\mathrm{L}, \mathrm{L}^{\prime}\right)$, as in the hypothesis of Alpern and Moeller (1977), then matches are generated in the appropriate regions of the $Y$ versus $R$ diagram. The open circles in Fig. 2 show intersection points for pairs of candidate protanomalous pigments and pairs of candidate deuteranomalous pigments. In the case of the candidate protanomalous pairs, the $\lambda_{\max }$ of $\mathrm{M}$ has been set at $534 \mathrm{~nm}$ and that of $\mathbf{M}^{\prime}$ has been displaced to succes- sively longer wavelengths. In the case of the deuteranomalous pigments, the $\lambda_{\max }$ of $\mathrm{L}$ has been set at $560 \mathrm{~nm}$ and that of $\mathrm{L}^{\prime}$ has been successively displaced to shorter wavelengths. If optical density is held constant (in this case, at 0.3) and the absolute separation of the candidate protanomalous pigments $M$ and $\mathbf{M}^{\prime}$ is not large, each shift of $1 \mathrm{~nm}$ in the value of $\mathbf{M}^{\prime}$ corresponds to a change of about 0.5 in $R$ and 0.2 in $Y$. For deuteranomalous pairs (L, $\mathrm{L}^{\prime}$ ), each shift of $1 \mathrm{~nm}$ in $\mathrm{L}^{\prime}$ corresponds to a change of approximately 0.7 in the $R$ value (and very little change in $Y$ ).

\section{Determination of the photopigments that are satisfied by a particular Rayleigh match}

For a given optical density, there are a maximum of two $\lambda_{\max }$ values that yield pigments satisfied by a particular Rayleigh match. Fig. 3 shows how the difference in excitation in a photoreceptor caused by a Rayleigh match setting of $R=40$ and $Y=15$ (i.e. a normal match) changes with the wavelength of peak sensitivity and the optical density of the photopigment. Where the curves intersect a horizontal line passing through zero on the ordinate, there is no difference in excitation caused by the mixing and reference light. Hence, a photopigment of this peak sensitivity (abscissa) will be satisfied by the Rayleigh match settings.

Fig. 3 makes clear that it is not possible to identify a unique pair of pigments that satisfy a given Rayleigh match. For the match is determined not only by the $\lambda_{\max }$ of the photopigment but also by its optical density (He \& Shevell, 1995). The Rayleigh match can be used only to predict the family of possible photopigments that may be present in the observer.

\section{Reconstruction of anomalous matches}

The matching loci derived in Fig. 2 for both forms of anomalous trichromacy do overlap with those found empirically (Hurvich, 1972). However, if the optical density is held at 0.3 - and if the pigment is described by the template of Baylor et al. (1987) — then

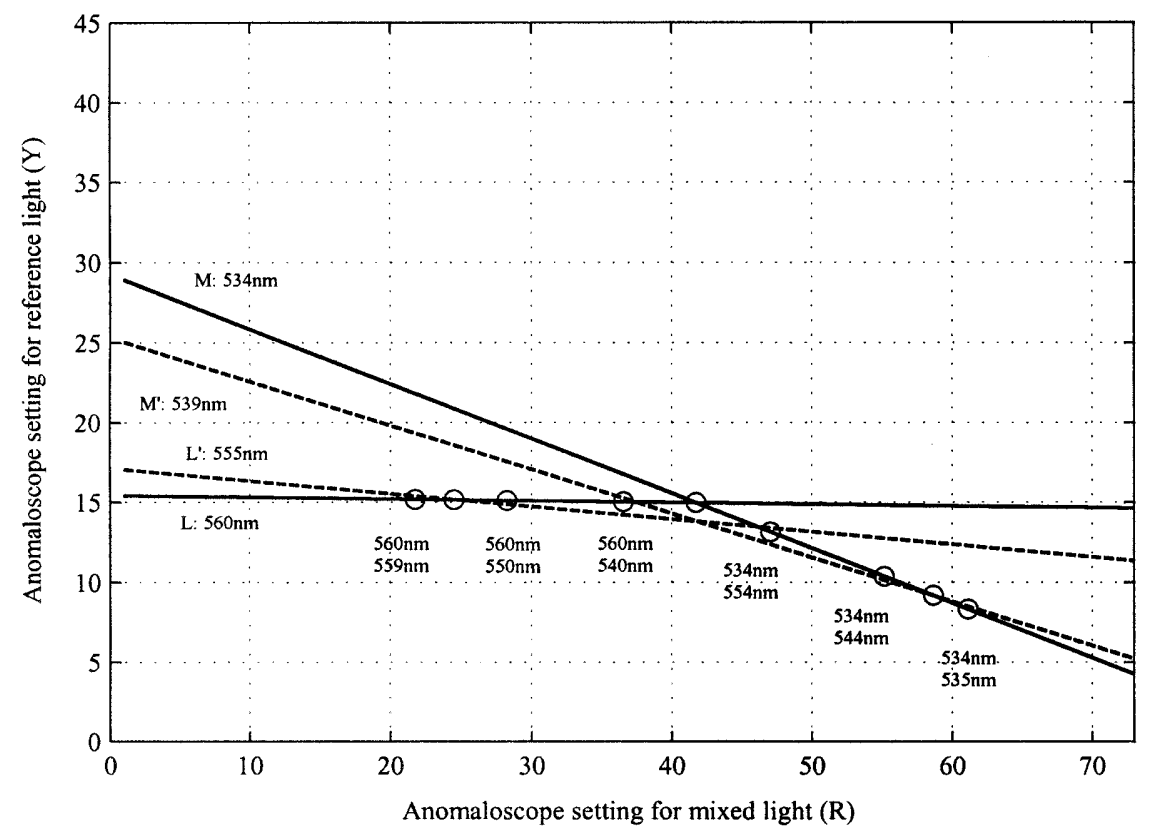

Fig. 2. Predicted match midpoints (circles) for a range of protanomalous, deuteranomalous and normal observers. Lines show examples of predicted loci for normal photopigments (solid lines) and photopigments that might be found in anomalous trichromats (dashed) in place of the normal $\mathrm{M}$ or $\mathrm{L}$ photopigments. 


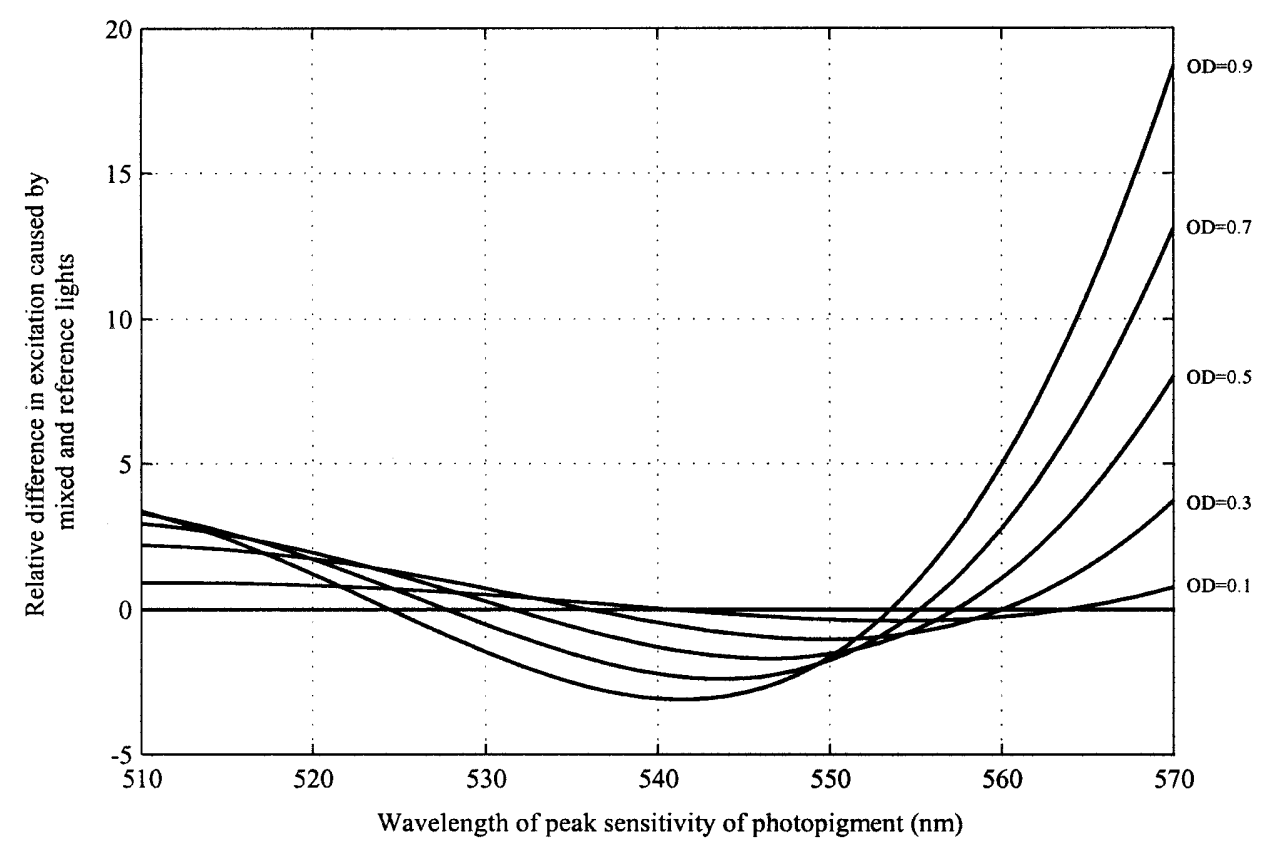

Fig. 3. Differences in photoreceptor excitation caused by the match $R=40$ and $Y=15$ for a range of pigments with different values of $\lambda_{\max }$ and of optical density.

it is not possible to generate deuteranomalous matches at $R$ values lower than 20. Yet classical studies show deuteranomalous observers who make matches as low as $R=15$ on the Nagel anomaloscope and who have a small matching range (Willis \& Farnsworth, 1952); and we ourselves have found subjects who exhibit such behavior on the Oculus anomaloscope (which is designed to simulate the Nagel). Such matches can be reconstructed, however, if optical density is allowed to vary. Fig. 4 shows predicted matches for a deuteranomalous observer (peak sensitivities at
$558 \mathrm{~nm}$ and $561 \mathrm{~nm}$ ) at a range of optical densities from 0 to 0.6. Matches can be made throughout the entire diagnostic range for deuteranomaly without altering peak sensitivities of the two photopigments.

A long-standing mystery is that there is no strong relationship between the midpoints and the ranges of deuteranomalous matches (Hurvich, 1972). If optical density is held constant, our model suggests that a strong relationship should be present: as the two deuteranomalous pigments increase in separation, the intersection

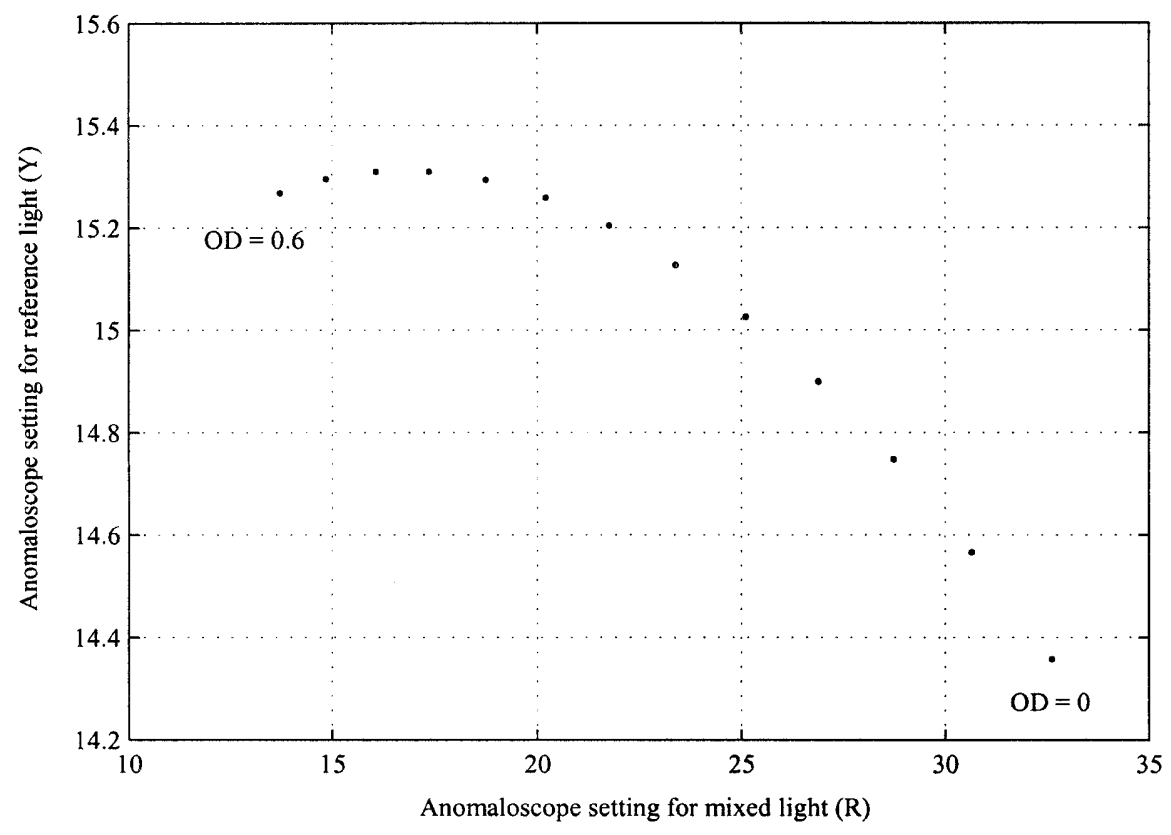

Fig. 4. Predicted match midpoints for a deuteranomalous observer (peak sensitivities at 558 and $561 \mathrm{~nm}$ ) with both photopigment optical densities ranging from 0 to 0.6 in increments of 0.05 . 
of the individual loci occurs at higher values of $R$ and the angle of intersection increases (Fig. 2); and it is reasonable to expect that matching range would be reduced as the angle of intersection increased. One interesting possibility is that an empirical relationship between match midpoint and range is masked by variation in optical density (He \& Shevell, 1995), which can independently change the slope of the locus of each pigment (Fig. 1B). For the matches of Fig. 4, the angle of intersection actually increases as the match moves away from that of a normal subject, rising from $2.3 \mathrm{deg}$ for an optical density of 0 to $2.75 \mathrm{deg}$ for an optical density of 0.6. This should relate to some decrease in matching range even though the match has moved further into the deuteranomalous region by about $19 R$ units. So, if photopigment optical density is allowed to vary in a population, any relationship between matching midpoint and range could be masked.

\section{Pseudoprotanomaly}

A number of conditions, such as central serous choroidopathy and choroideremia, produce the condition known as pseudoprotanomaly, in which the midpoint of the Rayleigh match is shifted to higher values of $R$ (Smith et al., 1978). Showing that pseudoprotanomaly is often associated with (1) a reduced effect of area on the match and (2) a broadening of the Stiles-Crawford effect, Pokorny et al. (1980) have convincingly argued that pseudoprotanomaly arises from a reduction in the effective optical density of the cones, and that this reduction is secondary to disordered alignment of the cones. In Fig. 5, we have used the template of Baylor et al. (1987) to simulate the trajectory of matches obtained for a pair of normal photopigments $\left(\lambda_{\max }=534 \mathrm{~nm}, 561 \mathrm{~nm}\right)$ as the optical density of both pigments is reduced, in the range $0.3-0.01$. This simulation suggests that pseudoprotanomaly could displace matches to at least $R=50$ on the Oculus anomaloscope. However, the trajectory for reduced optical density is shallower than the trajectory obtained by holding one pigment at $\lambda_{\max }=$
$534 \mathrm{~nm}(\mathrm{OD}=0.3)$ and reducing the $\lambda_{\max }$ of the second pigment-a trajectory simulating conventional protanomaly.

\section{Discussion}

Rayleigh matches have often been modelled previously. The one advantage of using a photopigment template, as in the present study, is that the general behavior of the match can be tracked as the $\lambda_{\max }$ and the optical density of the candidate photopigments are systematically altered. The model is dependent on the accuracy of the particular template adopted, and indeed on the underlying assumption that the absorption spectra of opsins are constant in form when plotted on an abscissa of log frequency. However, although some uncertainty must attend the prediction of specific matches, the use of the template makes visible the general behavior of matches as the absorption spectra of the underlying pigments are altered.

We have seen that it is usually not possible, from a given Rayleigh match, to predict the photoreceptor properties of an individual observer, because different combinations of peak sensitivity and optical density may give the same match. However, a consideration of the matching loci of individual photopigments suggests how the candidate photopigments could be narrowed down. Whereas a given match is not unique to a particular set of photopigments, the change of that match under bleaching may be unique. Since bleaching can be modelled by a change in optical density, the model can be used to predict the trajectory of Rayleigh matches that will occur for any set of photopigments under bleaching by any spectrally defined light. Performance under such conditions can therefore be used to narrow down the possible combinations of photopigment optical density and peak sensitivity possessed by the observer (Wyszecki \& Stiles, 1980; Burns \& Elsner, 1993). This could be of value in obtaining an approximate description of the photoreceptor properties of an observer using equipment no more specialized than an anomaloscope and a light source of known spectral composition. There are other operations

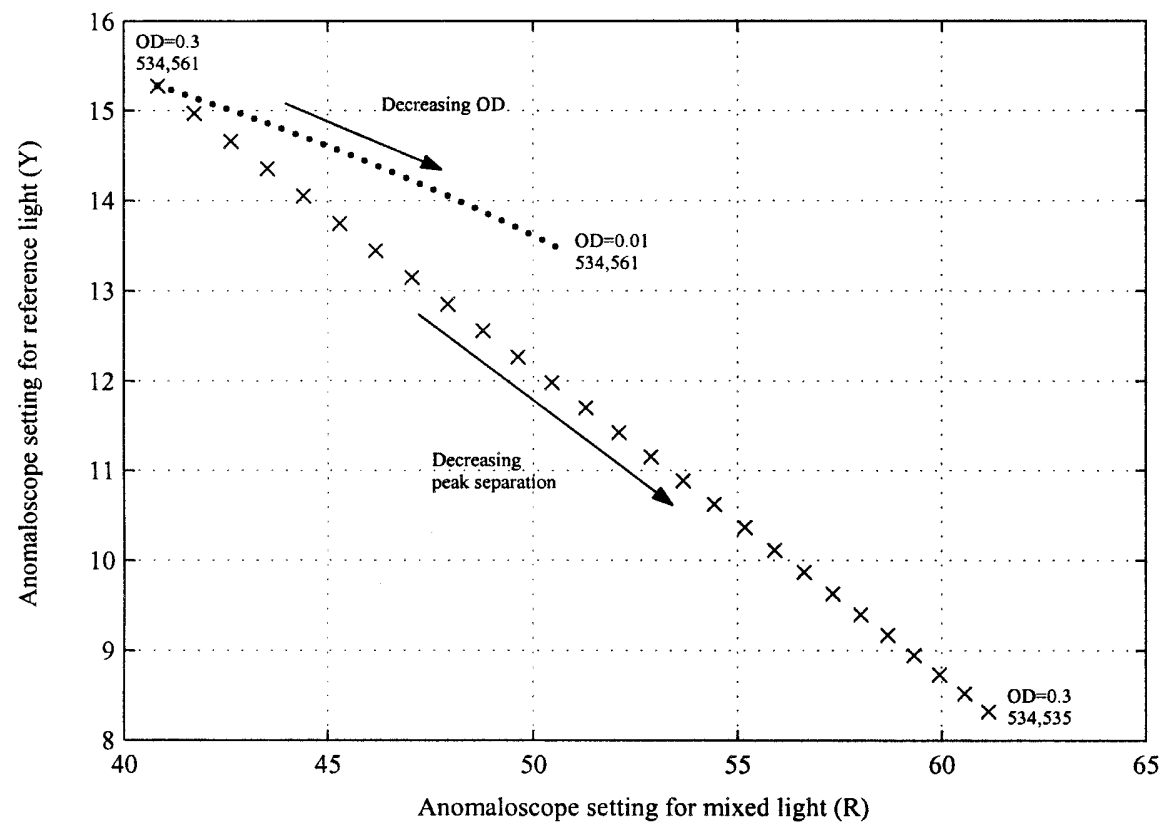

Fig. 5. A comparison of the loci of protanomalous and pseudoprotanomalous matches. Matches predicted with decreasing optical density of normal photopigments are shown with dots, while matches made by decreasing peak separation are shown with crosses. 
that alter effective optical density, such as an increase in stimulus area, and these similarly could be used to generate a trajectory.

\section{Acknowledgments}

Peter B.M. Thomas was supported by the Medical Research Council and the Isaac Newton Trust.

\section{References}

Alpern, M. (1979). Lack of uniformity in colour matching. Journal of Physiology 288, 85-105.

Alpern, M. \& Moeller, J. (1977). The red and green cone visual pigments of deuteranomalous trichromacy. Journal of Physiology 266, 647-675.

Alpern, M. \& WAKE, T. (1977). Cone pigments in human deutan colour vision defects. Journal of Physiology 266, 595-612.

Alpern, M., Fulton, A.B. \& Baker, B.N. (1987). "Self-screening" of rhodopsin in rod outer segments. Vision Research 27, 1459-1470.

BAKER, H.D. (1966). Single-variable anomaloscope matches during recovery from artificial red blindness. Journal of the Optical Society of America 56, 686-689.

BAylor, D.A., NunN, B.J. \& Schnapf, J.L. (1987). Spectral sensitivity of cones of the monkey Macaca fascicularis. Journal of Physiology 390, $145-160$.

Bone, R.A., LANDRUM, J.T. \& CAIns, A. (1992). Optical density spectra of the macular pigment in vivo and in vitro. Vision Research 32, 105-110.

BRINDLEY, G.S. (1953). The effects on colour vision of adaptation to very bright lights. Journal of Physiology 122, 332-350.

Burns, S.A. \& Elsner, A.E. (1993). Color matching at high illuminances: Photopigment optical density and pupil entry. Journal of the Optical Society of America A 10, 221-230.

He, J.C. \& SHevell, S.K. (1995). Variation in color matching and discrimination among deuteranomalous trichromats: Theoretical implica- tions of small differences in photopigments. Vision Research 35 2579-2588.

Hurvich, L.M. (1972). Color vision deficiencies. In Visual Psychophysics, Vol. 7/4, ed. Jameson, D. \& Hurvich, L.M., pp. 582-624. Berlin: Springer-Verlag.

Merbs, S.L. \& Nathans, J. (1992). Absorption spectra of the hybrid pigments responsible for anomalous color vision. Science 258, 464-466.

Mitchell, D.E. \& Rushton, W.A. (1971a). The red-green pigments of normal vision. Vision Research 11, 1045-1056.

Mitchell, D.E. \& Rushton, W.A. (1971b). Visual pigments in dichromats. Vision Research 11, 1033-1043.

Pokorny, J. \& SMith, V.C. (1976). Effect of field size on red-green color mixture equations. Journal of the Optical Society of America 66, 705-708.

Pokorny, J., SMith, V.C. \& ERnEST, J.T. (1980). Macular colour vision defects: Specialized psychophysical testing in acquired and hereditary chorioretinal diseases. International Ophthalmology Clinics 20, 53-81.

RAYleigh, L. (1881). Experiments on colour. Nature 25, 64-66.

Rushton, W.A. (1972). Pigments and signals in colour vision. Journal of Physiology 220, 1-31P.

Smith, V.C., Pokorny, J. \& Diddie, K.R. (1978). Color matching and Stiles-Crawford effect in central serous choroidopathy. Modern Problems in Ophthalmology 19, 284-295.

Stockman, A., Sharpe, L.T. \& Fach, C. (1999). The spectral sensitivity of the human short-wavelength sensitive cones derived from thresholds and color matches. Vision Research 39, 2901-2927.

Willis, M.P. \& Farnsworth, D. (1952). Comparative Evaluation of Anomaloscopes, Report \#190, pp. 1-89. U.S. Naval Submarine Base, New London.

Winderickx, J., Battisti, L., Hibiya, Y., Motulsky, A.G. \& Deeb, S.S. (1993). Haplotype diversity in the human red and green opsin genes: Evidence for frequent sequence exchange in exon 3. Human Molecular Genetics 2, 1413-1421.

Wyszecki, G. \& Stiles, W.S. (1980). High-level trichromatic color matching and the pigment-bleaching hypothesis. Vision Research 20, 23-37. 